\title{
5. Schlussbemerkung
}

Das Ziel dieser Arbeit bestand in der Ermittlung von nachfragerseiten Auswirkungen der Kundenintegration - positiver und negativer Art - sowie der näheren Analyse der Wirkungsbeziehung zwischen der Kundenintegration und der Kundenzufriedenheit. Resümierend kann dabei festgehalten werden, dass die vorliegende Studie einen nicht unwesentlichen Beitrag zur Auswirkungsforschung hinsichtlich der Kundenintegration darstellt. Die empirische Untersuchung konnte eine Reihe bislang teils unerforschter, nachfragerseitiger Auswirkungen der Kundenintegration identifizieren. Besonders signifikant zeigten sich dabei die positiven Auswirkungen der Unsicherheitsreduktion sowie des Enjoyment, wobei hinsichtlich der negativen Auswirkungen der im Zuge der Kundenintegration entstehende logistische Aufwand sowie eine damit einhergehende signifikante Reduktion der Zahlungsbereitschaft herausragen. Die Ambivalenz bzw. die Zweischneidigkeit des Konzepts der Kundenintegration wurde deutlich, wenn auch nur bedingt: Während einige negative Effekte der Kundenintegration erkannt wurden, konnten diesen jedoch keine negativen Auswirkungen auf die Kundenzufriedenheit nachgewiesen werden, was durchaus als Limitation der Studie betrachtet werden kann. Dem gegenüber steht jedoch die bedeutsame Reduzierung der kundenseitigen Zahlungsbereitschaft. Es wird ersichtlich, dass es bei der Ausgestaltung des Integrationsmanagements eine Vielzahl von Faktoren zu berücksichtigen sind, welche sich über die Kundenzufriedenheit in Form der Kundenbindung indirekt auf das Nachfragerverhalten auswirken. Um die Integration demnach zu optimieren, muss eine gesamtheitliche, beidseitige Sichtweise eingenommen werden, wofür die Durchführung einer dyadischen Untersuchung geeignet wäre. In der Ermittlung eines beidseitigen Optimums der Kundenintegration besteht ein Ansatzpunkt für künftige Forschungsbeiträge. Nur, wenn auch kundenseitige Implikationen der Ausgestaltung von Kundenintegrationsmaßnahmen betrachtet werden, kann ein effektives, kundengerichtetes Integrationsmanagement betrieben werden und die Mitwirkung des Nachfragers in Leistungserstellungsprozessen für beide Seiten - sowohl für Nachfrager, als auch für Anbieter Früchte tragen, worin sowohl die Herausforderung, als auch die Ambivalenz des Konzeptes besteht.

Es gilt folglich für die Anbieter, bereits bei der Ausgestaltung von Kundenintegrationsmaßnahmen darauf zu achten, dass diese nicht nur der internen, betrieblichen Produktivitäts- und Kostenoptimierung dienen, sondern auch den Nutzen des Kunden fördern bzw. seine Zufriedenheit gewährleisten, respektive erhöhen - denn nur zufriedene Kunden sind langfristig auch gute Kunden. 